\title{
Desarrollo computacional de piezas de fachada ventilada para la mejora de su respuesta a flexión
}

\author{
BEATRIZ DEFEZ ${ }^{1}$, GUILLERMO PERIS-FAJARNÉS ${ }^{1}$, ISMAEL LENGUA ${ }^{1}$, VÍCTOR SANTIAGO-PRADERAS ${ }^{1}$, JOSÉ MANUEL SORIA ${ }^{2}$ \\ ${ }^{1}$ Centro de Investigación en Tecnologías Gráficas, Universitat Politècnica de València. Camino de Vera s/n-8L, 46022 Valencia, España \\ ${ }^{2}$ Cerámica Mayor S.L. Partida Planet s/n, Callosa d'En Sarria, 03510 (Alicante) \\ bdefez@degi.upv.es
}

\begin{abstract}
La fachada cerámica ventilada es un producto en auge en el sector de la construcción actual. Para cubrir los requerimientos del mercado sin perder competitividad, es necesario desplegar técnicas de diseño que permitan fabricar nuevos modelos con mejores prestaciones mecánicas a menor coste y ciclos de desarrollo más rápidos. En este trabajo se desarrolla una metodología de diseño geométrico de fachadas ventiladas destinada a incrementar la resistencia a flexión de las piezas, basada en modelado asistido por ordenador y simulación por elementos finitos. La resistencia a flexión es el indicador comúnmente usado para medir la resistencia mecánica de las fachadas, y su valor debe cumplir con los mínimos establecidos en la norma de calidad en la fabricación ISO 10545. En el desarrollo de la metodología se aborda con detalle la obtención experimental de las propiedades del material empleado, y la adecuación de ciertas simplificaciones necesarias para una simulación útil. Finalmente, la metodología ha sido validada experimentalmente.
\end{abstract}

Palabras clave: fachada ventilada, fuerza de rotura a flexión, análisis por elementos finitos.

\section{INTRODUCCIÓN}

La fachada cerámica ventilada es un producto en auge en el sector de la construcción actual. Para cubrir las necesidades del mercado sin perder competitividad es necesario desarrollar técnicas de diseño que permitan fabricar nuevos modelos de mejores prestaciones a menor coste y ciclos de desarrollo más rápidos [1-4]. En este sentido, el desarrollo de metodologías para la aplicación de CAD (del inglés "Computer Aided Design") al desarrollo de fachadas ventiladas puede ser una solución muy interesante.

En este trabajo se desarrolla una metodología de diseño geométrico y evaluación de piezas individuales de fachada ventilada frente a esfuerzos de flexión basada en CAD y simulación por elementos finitos. La experiencia de la empresa colaboradora en el estudio demuestra que la resistencia a flexión es el parámetro mecánico determinante en la fabricación eficiente de fachadas ventiladas cerámicas, por cuanto representa mejor que ninguna otra característica a la resistencia mecánica de la pieza. Además, el valor medio de la resistencia a flexión calculado sobre una muestra de fabricación debe cumplir con la norma de calidad en la fabricación ISO 10545-4 [5] ineludiblemente.

El modelado tridimensional es una técnica conocida en el diseño de materiales cerámicos [6], pero relativamente nueva en el sector de las baldosas cerámicas en su conjunto, debido al uso mayoritario del diseño bidimensional. Por su parte, el análisis por elementos finitos ha sido ampliamente utilizado en el campo de las cerámicas avanzadas, pero sólo ocasionalmente en el campo de los pavimentos y revestimientos. Por otro lado, estas escasas aplicaciones se han centrado en modelos bidimensionales, siendo las simulaciones térmicas las más recurrentes. Así, la mayoría de estudios han dejado de lado el modelado tridimensional y las exigencias mecánicas [7]. Sin embargo, estudios más recientes ya han utilizado tanto el modelado tridimensional como el análisis de elementos finitos para el estudio de la optimización del diseño de las baldosas cerámicas a partir de sus características mecánicas [8-9].

En el desarrollo de la metodología que resume este trabajo, se aborda con detalle la obtención experimental de las propiedades del material empleado, y la adecuación de ciertas simplificaciones necesarias para un modelado de la fachada ventilada cerámica y una simulación de su ensayo de flexión útil. Posteriormente, la metodología ha sido validada experimentalmente.

\section{RESUMEN DE LA METODOLOGÍA DE TRABAJO}

El esquema de la investigación desplegado en este trabajo se muestra en la figura 1.

Se seleccionó una geometría de pieza de fachada ventilada y un material cerámico para su manufactura representativos de la producción usual. A continuación se fabricó un lote piezas de este modelo con los medios productivos habituales. Las piezas de este lote fueron sometidas al ensayo normalizado de flexión, según especifica la norma ISO 10545-4, obteniendo un valor promedio de su fuerza de rotura a flexión $(\mathrm{F})$.

En paralelo, se obtuvieron las propiedades físicas del material cerámico del estudio. Contando con los valores reales 


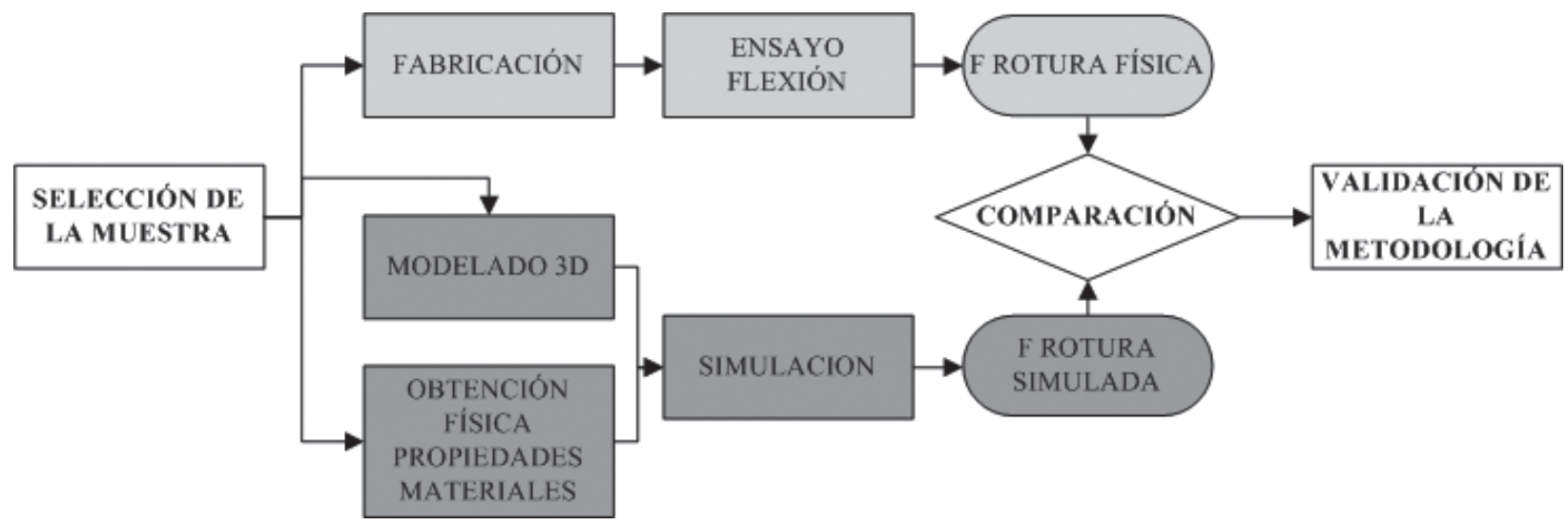

Figura 1. Esquema del desarrollo de la metodología

de esas propiedades, se modeló la geometría elegida mediante un programa CAD. En este mismo entorno de modelado, se reprodujeron de forma virtual las condiciones del ensayo a flexión, y se ejecutó la simulación, obteniendo una fuerza de rotura simulada $\left(\mathrm{F}_{\mathrm{S}}\right)$.

La comparación de ambas fuerzas de rotura permitió determinar la validez de la metodología de modelado y simulación planteada. Cuanto más similares fueran estas fuerzas, más adecuada sería la metodología.

\section{PROCEDIMIENTO FÍSICO}

\subsection{Muestras}

La pieza de fachada ventilada escogida para la investigación fue la conocida como "Modelo FK", fabricada por la empresa Cerámica Mayor S.L. Esta pieza tiene unas dimensiones de 300 $\mathrm{mm}$ x $600 \mathrm{~mm}$ x $16 \mathrm{~mm}$. Una representación de la pieza puede verse en la figura 2 :

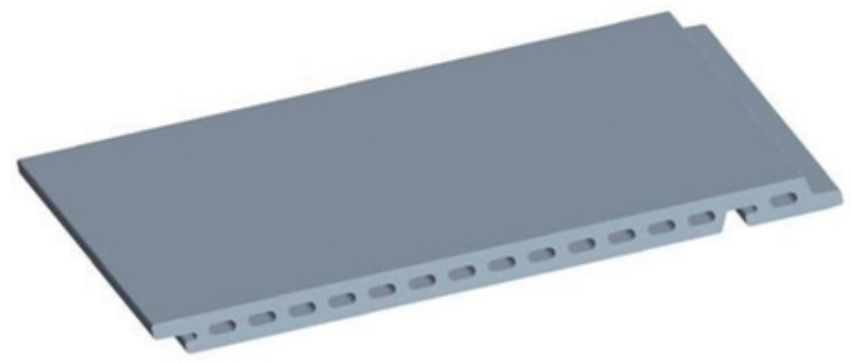

Figura 2. Fachada ventilada empleada en el estudio

El material cerámico utilizado para la fabricación de esta pieza fue una pasta blanca de porcelánico utilizada habitualmente en la empresa para este modelo. La composición de esta pasta fue $\mathrm{SiO}_{2} 51,0 \%, \mathrm{Al}_{2} \mathrm{O}_{3} 19,0 \%, \mathrm{Fe}_{2} \mathrm{O}_{3} 0,5 \%, \mathrm{CaO}$ $7,0 \%, \mathrm{MgO} 5,2 \%, \mathrm{NaO}_{2} 0,4 \%, \mathrm{~K}_{2} \mathrm{O} 3,7 \%, \mathrm{TiO}_{2} 0,7 \%$ y Chamota $12,5 \%$.

\subsection{Ensayo de flexión}

La parte 4 de la norma ISO 10545 establece el método normalizado para la determinación de la fuerza de rotura y resistencia a flexión de las baldosas cerámicas. En el caso de baldosas extruidas se coloca la probeta de manera que las estrías de extrusión sean perpendiculares a los rodillos de apoyo.

La fuerza aplicada que se puede leer en la máquina de flexión en el momento de la rotura de la pieza recibe el nombre de fuerza o fuerza de rotura a flexión (F).

El número mínimo de probetas para la realización del ensayo viene determinado por el tamaño de las piezas. Para una dimensión $\geq 48 \mathrm{~mm}$ se deben emplear un mínimo 7 piezas. En esta investigación, se fabricó un lote de 70 piezas de las características ya comentadas para someterlo al ensayo de flexión.

Los ensayos fueron llevados a cabo en una máquina de ensayos electromecánica MEM-101/SDC ${ }_{(\mathrm{v} 1.1)}$ equipada para la realización de ensayos de flexión.

\section{PROCEDIMIENTO VIRTUAL}

\subsection{Determinación de las propiedades mecánicas del material}

Para la realización de la simulación numérica es necesario caracterizar el material a partir del cual se fabrican las piezas de fachada ventilada. Los valores concretos usados fueron: densidad $\rho=2257 \mathrm{Kg} / \mathrm{m}^{3}$; resistencia a tracción $\sigma_{\mathrm{T}}=25.17 \mathrm{~N} /$ $\mathrm{mm}^{2}$; módulo de Young E=22553 N/ mm²; y coeficiente de Poisson $\mu=0.20$.

El modulo de Young o módulo de elasticidad del material se obtuvo experimentalmente a partir del ensayo de flexión. Teniendo en cuenta la mecánica clásica de materiales formulada por Newton y Hooke [10], se deduce que hay una relación lineal entre la fuerza aplicada a la pieza cerámica y la flecha generada en su sección transversal cuando se realiza este ensayo. De esta relación se puede extraer el valor del módulo de Young según la ecuación [11-12]:

\section{$\mathrm{E}=\mathrm{FL}^{3} /\left(4 \mathrm{fbh}^{3}\right)$}

Donde E es el modulo de Young; L es la distancia entre apoyos; F es la fuerza aplicada o fuerza de rotura en última instancia; f es la flecha o deformación central; b es el ancho y $\mathrm{h}$ es el grosor de la baldosa.

Se realizó el ensayo de flexión sobre un lote de piezas cerámicas fabricadas con del material del estudio. Este lote 
estaba compuesto por 70 piezas de baldosa simple (forma en paralelepípedo) de tamaño 310 × $310 \times 9.7 \mathrm{~mm}$; y 100 piezas de baldosa simple de tamaño 245 × 245 × $9.1 \mathrm{~mm}$. Se consideraron dos formatos distintos para comprobar que el tamaño de las probetas no tenía una influencia significativa en la obtención del módulo. El valor promedio de E obtenido fue de 22553 N/ $\mathrm{mm}^{2}$.

El valor del coeficiente de Poisson fue tomado de la bibliografía, dado que su valor no varía en demasía entre las distintas composiciones de cerámica de pasta blanca y su obtención experimental no era sencilla con el equipamiento disponible. Se decidió tomar el valor de $0.20 \mathrm{~m} / \mathrm{m}$ de Bowman y Banks [13] puesto que el material empleado en el citado trabajo tenía una composición similar al empleado en esta investigación.

\section{DISEÑO DE LA SIMULACIÓN}

\subsection{Modelado de las piezas}

Para el modelado de la pieza de fachada ventilada escogida, y su posterior análisis por elementos finitos, se utilizó un software comercial de diseño tridimensional y simulación.

En el modelado se tuvo en cuenta el proceso de fabricación real, es decir, la extrusión. Se creó el perfil transversal de la pieza en dos dimensiones y se aplicó la función de extrusión para obtener el cuerpo tridimensional final.

\subsection{Simulación del ensayo de flexión}

En la simulación del ensayo de flexión, se buscó reproducir las condiciones reales del ensayo lo más fielmente posible. Por otro lado, la simulación debía ser operativa, es decir, su complejidad no debía exceder la capacidad del programa y del equipo para realizar los cálculos numéricos, y los resultados debían ser concluyentes. Conjugando estos dos condicionantes, se realizaron las siguientes simplificaciones.

\subsubsection{SIMPLIFICACIÓN DEL RAYADO DE EXTRUSIÓN}

Las piezas extruidas cuentan con un relieve en su superficie inferior conocido como "rayado de extrusión". El rayado de los productos del estudio apenas tenía $1 \mathrm{~mm}$ de profundidad, por lo que su influencia en el comportamiento a flexión debía ser, a priori, mínima. Sin embargo, el rayado, incluso con tan poca profundidad, interfiere en el análisis por elementos finitos como un potente concentrador de tensiones. Además aumenta exponencialmente el número de elementos que deben utilizarse para el análisis, lo que incrementa hasta valores poco prácticos el tiempo de cálculo computacional.

Tras estas consideraciones sobre el rayado, se decidió eliminar el rayado del modelo virtual. Por tanto, la superficie posterior del modelo virtual es plana. Esta superficie simplifica sensiblemente el cálculo de la fuerza de rotura a flexión simulada y reduce el tiempo de ejecución del análisis.

\subsubsection{SIMPLIFICACIÓN DE LOS APOYOS}

Para reducir el número de objetos presentes en la simulación, los cilindros de apoyo y el cilindro de aplicación de la fuerza fueron sustituidos por dos líneas de acción ubicadas sobre la propia pieza cerámica. Estas líneas simulan el contacto de los cilindros con la pieza.
Se consideraron líneas en lugar de superficies, debido al hecho de que los cilindros de la máquina de flexión utilizada en este trabajo no se encontraban recubiertos con caucho. Siendo puramente metálicos, no se consideró que sufrieran deformación apreciable al aplicar la fuerza sobre la pieza.

Las líneas de apoyo se colocaron en la superficie inferior de la pieza a $10 \mathrm{~mm}$ del borde, mientras que la línea de aplicación de la fuerza se situó en la superficie superior en posición central equidistante a las líneas de apoyo. Para simular las condiciones de contorno reales se restringió el movimiento vertical en los apoyos (eje vertical Z). Además, para facilitar la convergencia en el cálculo computacional, fue necesario añadir una restricción adicional a la línea de aplicación de la fuerza, impidiendo el movimiento en horizontal de la misma (movimiento según los ejes del plano X e Y, coplanarios con la superficie de apoyo de la pieza).

\subsubsection{SIMPLIFICACIÓN DE LA APLICACIÓN DE LA FUERZA}

Según las pautas del ensayo de flexión normalizado, la fuerza debe ser repartida uniformemente en el rodillo central, de forma que el esfuerzo aumente en $1 \pm 0.2 \mathrm{~N} / \mathrm{mm}^{2}$.

El modelado de una fuerza dinámica añade complejidad a la simulación. Así, se plantea la conveniencia de considerar una aplicación instantánea (o estática) de la fuerza. Para admitir esta simplificación, se estudió la influencia de la velocidad de aplicación de la fuerza en los resultados de resistencia a flexión.

Como en el caso de la determinación de E, se realizó el ensayo de flexión sobre un lote de piezas cerámicas fabricadas con del material del estudio. Este lote estaba compuesto por 80 piezas de baldosa simple (forma en paralelepípedo) de tamaño $310 \times 310 \times 9.7 \mathrm{~mm}$.

Se formaron 8 sublotes de 10 piezas y se ensayaron a diferentes velocidades de aplicación de la fuerza. Los resultados promedio de $\mathrm{F}$ se presentan en la tabla I.

Tabla I. VARIación de F CON SU VElOCidad de APLiCaCión. PIEZAS DE BALDOSA SIMPLE Y MATERIAL DEL ESTUDIO

\begin{tabular}{|c|c|}
\hline $\begin{array}{c}\text { Velocidad de aplicación de la } \\
\text { fuerza (N/s) }\end{array}$ & F (N) \\
\hline 10 & 1653 \\
\hline 50 & 1708 \\
\hline 100 & 1688 \\
\hline 150 & 1639 \\
\hline 200 & 1542 \\
\hline 250 & 1590 \\
\hline 300 & 1660 \\
\hline 500 & 1674 \\
\hline
\end{tabular}

Como puede observarse en la tabla I, no parece existir una relación entre la velocidad de aplicación de la fuerza y el resultado de la fuerza de rotura a flexión. Se puede concluir que para el material de este estudio, el efecto de la velocidad de aplicación de la fuerza es despreciable en el ensayo de flexión. Por consiguiente, se puede aceptar el empleo de una fuerza instantánea para la simulación del ensayo mediante elementos finitos. 


\subsection{Método de cálculo de la fuerza de rotura simulada}

La fuerza de rotura simulada $\left(\mathrm{F}_{\mathrm{S}}\right)$, es aquella fuerza de rotura definida tal y como establece la norma ISO 10545, para la cual el modelo virtual de las piezas rompe, una vez ejecutada la simulación del ensayo de flexión.

Sin embargo, el programa de cálculo considera que el material (cualquiera que se modele) es infinitamente elástico, es decir, mayores esfuerzos experimentados en la pieza darán lugar a mayores deformaciones, pero nunca se pasará al estado plástico, ni por tanto se producirá rotura visible de la pieza dentro de la propia simulación. Así pues, es necesario conocer a partir de qué esfuerzo surgido en la pieza se debe considerar que existe ruptura, aunque el programa no la muestre como tal.

Se debe elegir por tanto un determinado criterio de fractura. En este caso, dado la naturaleza frágil de la cerámica, se escogió el criterio de Rankine o de la tensión máxima principal. Adaptado al caso de este trabajo, este criterio establece que la pieza romperá cuando el esfuerzo máximo principal surgido en el objeto sobrepase la tensión máxima de rotura a tracción característica del material [10-14].

Considerando un comportamiento esencialmente lineal antes de la rotura, se puede calcular esta fuerza de rotura simulada como:

$$
\mathrm{F}_{\mathrm{S}=} \mathrm{F}_{\mathrm{AEF}}{ }^{*} \sigma_{\mathrm{T} /} \sigma_{\mathrm{AEF}}
$$

Donde $\mathrm{F}_{\mathrm{S}}$ es la fuerza de rotura simulada, $\mathrm{F}_{\mathrm{AEF}}$ es la fuerza aplicada en la simulación, $\sigma_{\mathrm{T}}$ es la resistencia a tracción del material, y $\sigma_{\mathrm{AEF}}$ es la tensión máxima principal obtenida en el análisis por elementos finitos con la fuerza aplicada.

Se eligió una fuerza aplicada de valor $\mathrm{F}_{\mathrm{AEF}}=2000 \mathrm{~N}$ ya que los ensayos físicos previos habían dado como resultado fuerzas de rotura a flexión próximas a este valor.

\section{RESULTADOS}

Los resultados de fuerza de rotura obtenidos en el ensayo de flexión fueron: fuerza de rotura real o física $\mathrm{F}=1855 \mathrm{~N}$, y fuerza de rotura simulada $\mathrm{F}_{\mathrm{S}}=1800 \mathrm{~N}$.

\section{CONCLUSIONES}

Los resultados obtenidos muestran como el valor promedio $\mathrm{F}$ es inferior a $\mathrm{F}_{\mathrm{S}}$. Se puede entender esta diferencia teniendo en cuenta que la pieza simulada presenta un comportamiento ideal, mientras que las piezas reales pueden presentar varios defectos de fabricación que hagan descender su respuesta mecánica.

En cualquier caso, la diferencia apenas llega a un $3 \%$, por lo que ambas fuerzas pueden considerarse equivalentes. Por consiguiente puede afirmarse que la metodología desplegada para el modelado de la pieza de fachada ventilada, y la simulación del ensayo de flexión mediante elementos finitos es correcta. Así, se podrán emplear las simulaciones aquí diseñadas para investigar geometrías innovadoras en la fachada ventilada, con el inherente ahorro de tiempo y recursos económicos que ello supone, en comparación al método de diseño tradicional.

\section{AGRADECIMIENTOS}

Estainvestigaciónhasidofinanciadaenelmarcodel proyecto “MODELADO SÓLIDO TRIDIMENSIONAL Y ANÁLISIS POR ELEMENTOS FINITOS PARA LA SIMULACIÓN DE LA LÍNEA DE PRODUCCIÓN CERÁMICA", referencia: PAID06-08-3162, subvencionado por la Universitat Politécnica de València, y por la empresa Cerámica Mayor, S.L. Resultados previos de este trabajo fueron expuestos en el LII Congreso de la Sociedad Española de Cerámica y Vidrio, celebrado en Burgos del 3 al 6 de octubre de 2012, a través de póster “METODOLOGÍA PARA LA EVALUACIÓN DE PIEZAS DE FACHADA VENTILADA MEDIANTE SIMULACIÓN NUMÉRICA".

Los autores expresan una vez más su agradecimiento a la empresa Cerámica Mayor por contribuir a este trabajo con sus materiales y equipos.

\section{REFERENCIAS}

1. G. Silva Moreno, F.J. Mira Peidro, “Sector Cerámico. Tradición e innovación", Qualicer 2008, Con 27 - Con 49 (2008).

2. G. Silva, V. Cantavella, A. R. García, E. Bou, A. Miralles, E. Uviedo, "Estudio de la eficiencia energética de fachadas ventiladas cerámicas", Qualicer2008, Con.27-Con.49 (2008).

3. Bartolomé, M., Batalla, P., Gallego. T., Mira, F.J., Palencia J.J., Pitarch, A.M., Ramón, J.J., Silva, G., “Cerámica para la arquitectura. Volumen 2. Fachadas ventiladas y pavimentos técnicos", editado por ASCER (2009).

4. I. Paricio Ansuategui, "La fachada ventilada con ladrillo cara vista" Trabajo patrocinado por Hyspalit y el colegio oficial de arquitectos de Aragón (2006).

5. ISO 10545-4:2004, “Baldosas cerámicas. Parte 4: Determinación de la resistencia a la flexión y de la fuerza de rotura", International Organization for Standardization (2004).

6. J. Mackerle, "Finite element modelling of ceramics and glass, an addendum - a bibliography (1998-2004)", Engineering Computations, 22, 3, 297-373 (2005).

7. B. Defez, G. Peris-Fajarnés, I. Tortajada Montañana, L. Dunai, “Estudio de la evolución y nuevas técnicas de análisis por elementos finitos aplicadas al diseño de pavimentos cerámicos", Bol. Soc. Esp. Ceram. Vidr., 48, 8, 273278 (2009).

8. B. Defez, G. Peris-Fajarnés, I. Tortajada, F. Brusola, S. Morillas, "Flexural strength of non-constant thickness ceramic floorings by means of the finite element method", Int. J. Appl. Ceram. Technol.; 7, 16, 235-247 (2010).

9. B. Defez, G. Peris-Fajarnés, I. Tortajada, L. Dunai, F. Brusola, “Optimal design of deep back relief in ceramic floorings by means of the finite element method", J. Ceram. Soc. Jpn.; 116, 9, 941-949 (2008).

10. J. Gere, "Timoshenko. Resistencia de Materiales", International Thomson Editores Spain (2002).

11. Norma Chilena 987 - Madera: determinación de las propiedades mecánicas - Ensayo de flexión estática en la madera (1989).

12. Instituto de Promoción Cerámica, información publicada en su página web: "http://www.ipc.org.es".

13. R.G. Bowman, P.J. Banks, "The crucial need for computer modelling of tiling systems" I World Congress on Ceramic Tile Quality, 1, 617-634 (1996).

14. M Romero, P. Museros, M.D. Martínez, A. Poy,"Resistencia de Materiales", Publicacions de la Universitat Jaume I, Castellón (2003). 